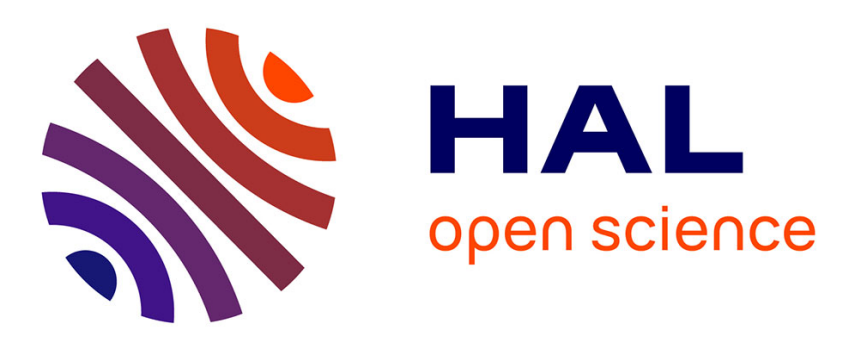

\title{
Control and data channels allocation for Large-Scale UWB-based WSNs
}

\author{
Jamila Ben Sliman, Ye-Qiong Song, Anis Koubâa
}

\section{To cite this version:}

Jamila Ben Sliman, Ye-Qiong Song, Anis Koubâa. Control and data channels allocation for LargeScale UWB-based WSNs. ComNet'09: The First International Conference on Communications, and Networking, Nov 2009, Hammamet, Tunisia. pp.64. inria-00435514

\section{HAL Id: inria-00435514 https://hal.inria.fr/inria-00435514}

Submitted on 24 Nov 2009

HAL is a multi-disciplinary open access archive for the deposit and dissemination of scientific research documents, whether they are published or not. The documents may come from teaching and research institutions in France or abroad, or from public or private research centers.
L'archive ouverte pluridisciplinaire HAL, est destinée au dépôt et à la diffusion de documents scientifiques de niveau recherche, publiés ou non, émanant des établissements d'enseignement et de recherche français ou étrangers, des laboratoires publics ou privés. 


\title{
Control and data channels allocation for Large-Scale UWB-based WSNs
}

\author{
Jamila Ben Slimane* ${ }^{* \ddagger}$ Ye-Qiong Song ${ }^{\ddagger}$ and Anis Koubaa ${ }^{\S \nsubseteq}$ \\ *Sup'Com-MEDIATRON, City of Communication Technologies, 2083 Ariana, Tunisia \\ ${ }^{\ddagger}$ LORIA and INPL, Campus Scientifique, BP 23954506 Vandoeuvre-les-Nancy, France \\ §IPP-HURRAY! Research Group, Polytechnic Institute of Porto, \\ Rua Antnio Bernardino de Almeida, 431, 4200-072 Porto, Portugal \\ 『Al-Imam Muhammad ibn Saud University, Computer Science Dept., 11681 Riyadh, Saudi Arabia \\ Email: jamilabs07@yahoo.fr, Song@loria.fr, akoubaa@dei.isep.ipp.pt
}

\begin{abstract}
Resource allocation is a critical issue for Wireless Sensor Networks (WSNs) especially for Ultra-Wide Band (UWB) based networks. In this paper, we present frequency allocation scheme for an efficient spectrum management that is able to statically assign control channels and dynamically reuse data channels for Personal Area Networks (PANs) inside a LargeScale WSN based on UWB technology. The frequency allocation problem is decomposed into two sub-problems: static control channel assignment and dynamic data channel allocation. The goal is two-folded: first, we aim to avoid congestion of control channel, second, to maximize simultaneous communications without suffering from interference in order to enhance throughput, decrease delay and minimize energy consumption.
\end{abstract}

\section{INTRODUCTION}

Wireless communication based on UWB signals has recently been quite attractive to the wireless community. Indeed, this emerging technology promises, high-rate, low power transmission, immunity to multi-path propagation and highprecision ranging capability. In this context, the recently standardized IEEE 802.15.4a protocol provides a new alternative to the physical layers of the IEEE 802.15.4 protocol, particulary developed for low-rate low-power Wireless Personal Area Networks (WPAN), among which one is based on the UWB technology. Nowadays multi-channel Media Access Control (MAC) protocols represent a critical topic of research in WSNs allowing parallel transmissions which can improve network performance. Both IEEE 802.15.4 and its recent amendment IEEE 802.15.4a standards allow dynamic channel allocation and use of multiple channels available at their physical layers but its MAC protocols are designed only for single channel to support different network architectures. Also, sensor's transceivers such as CC2420 used by current WSN hardware (MICAZ, TelosB, and CMU FireFly), provide multiple channels and as shown in [1] and [2] channel switch latency of CC2420 transceiver is short (just about $200 \mu s$ ). However, in the general case of dense Mesh WSNs, the exploitation of the multi-channel access is complex. This explains the imperative need to propose an adequate network architecture that can simplify and reduce the complexity of the resource sharing task in such networks and to design scalable and optimal multi-frequency MAC protocols. Given the several advantages offered by the UWB technology, we propose to investigate the
UWB physical layer specified by IEEE 802.15.4a standard for WSNs, by designing efficient schemes to ensure the best management of the UWB channels. Most currently deployed sensor networks use the same channel to communicate information towards nodes (control and data traffic) what leads to a complex problem of resource sharing especially in dense and large scale networks. Although multi-channel access protocols for WSNs are not new issues and have been a topic of research in such networks, proposed protocols are really very few. The principle of frequency allocation is firstly used in cellular networks like Global System for Mobile communications (GSM) [3]. In GSM networks, channel allocation is a function of network sizing (taking in account traffic distribution and subscriber model) and cellular planning, so the minimum distance of frequency reuse $D=\sqrt{3 K} \times R$ where $R$ represents the cell radius and $K$ represents the scale of cluster. However in WSNs, frequency reuse is a function of sensor's coverage, duty cycle of network's members and network's topology. In the literature, a number of multichannel MAC protocols have been proposed for WSNs [4]-[8]. The first multi-channel protocol, called Multi-frequency Media access control for wireless Sensor Networks (MMSN) [4], represents four frequency assignment schemes for WSN: exclusive frequency assignment, even selection, eavesdropping and implicit-consensus. The first scheme is used when the number of available frequencies is at least as large as the two-hop node number, it allocates channels in such a way that nodes within two hops are assigned different frequencies. Due to several broadcasts, the communication overhead in this scheme is relatively high. The second scheme ensures smaller overhead but it requires more physical frequencies that is, it assumes that frequency resource are abundant. Given that the two other schemes do not guarantee the assignment of different frequencies to two-hop neighbors, potential conflicts can not be avoided. Moreover, we note that MMSN's schemes allocate channels in static way which limits channel utilities as a node does not permanently use its assigned channel. In [5] and [6], the authors proposed a dynamic channel allocation based on agreement established between each sender and receiver nodes. Such approach may be suitable in light network but in dense network frequency negotiation messages can 
involve a considerable unnecessary overhead (e.g negotiation message retransmissions following their reception failure). The advantage of those protocols is the use of several channels for control traffic which can avoid control channel congestion problem. All previous multi-channel allocation schemes are proposed for classical WSNs operating on $2.4 \mathrm{Ghz}$ band without any support of quality-of-service $(\mathrm{QoS})$ mechanisms, however the authors in [7] proposed the first Multi-Channel MAC protocol (MCMAC) taking into account the notion of priority during channel allocation process inside a cluster. In [8], the authors proposed the first multi-channel scheme designed for UWB based IEEE 802.15.3 networks.

Our paper makes the following main contributions.

- First, to reduce the complexity of channels sharing in dense and large-scale full Mesh WSN, we focus on network architecture proposed in [9]. Then, we take advantage from some notions of graph theory but in a new context which was not previously addressed. That must obey, on the one hand, to the conditions of resource sharing relative to WSNs (which are different to the resource allocation conditions of other wireless networks like GSM) and, on the other hand, by completely exploiting advantages offered by the UWB technology.

- Second, To avoid the congestion of control channel, we propose to statically assign the optimal number of control channels to ensure one control channel per PAN.

- Finally, to maximize the number of simultaneous communications per active PAN, we try to dynamically assign data channels according to the spatial channel reuse and the duty cycle's information of PANs.

The rest of the paper is organized as follows: In section 2, we present the system model. In section 3, we detail the static channel allocation scheme. Section 4 presents the dynamic data channel allocation scheme. In section 5, we evaluate the proposed schemes by analyzing and commenting some results.

\section{SYSTEM MODEL}

\section{A. Network Topology}

In order to deploy a dense network supporting a considerable number of nodes, we proposed in [9] a three-tiered network to represent the global network, using UWB sensors in the first and second network levels. We have chosen the UWB technology for the following raisons:

- its extremely low transmitting power minimizing interference,

- high data rates allowing real time and high data rate applications,

- location capacity ensuring mobility management and node identification.

For the third tier, we propose Wifi network to benefit from its high data rate, large coverage and security aspects. We aim to design a WHSN (Wireless Hospital Sensor Network) for an application in hospital (medical monitoring of patients and management of doctors) but the proposed system model as well as channel allocation schemes to be proposed can apply to more general WSNs contexts. Fig. 1 shows all network layers composing the WHSN.

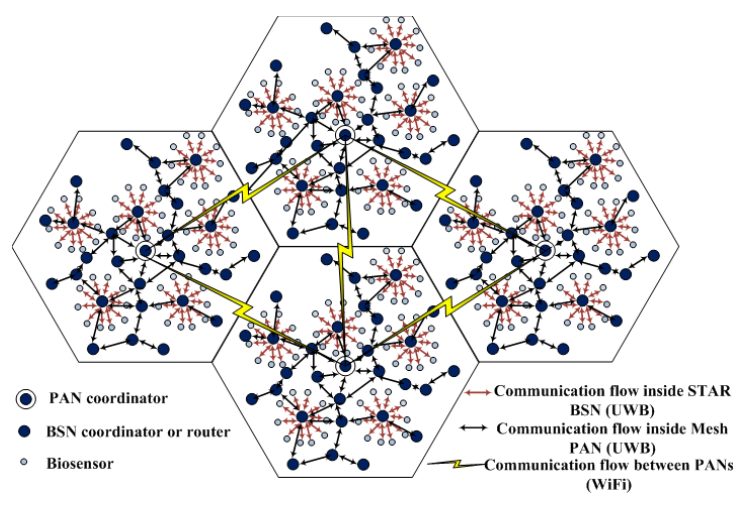

Fig. 1. WHSN architecture

The lowest level represents the Body Sensor Network (BSN). We can model an elementary BSN by a star network composed of one coordinator and a set of biosensors that ensure physiological measurements and medical monitoring of patient. To improve patient's network performance in a dense hospital environment, we propose overlaying the network of BSNs with a second upper level network or PAN. As shown in Fig. 1, the network is represented by a hexagonal cell of sensors organized in mesh topology including one PAN coordinator, several mobile BSNs coordinators (one coordinator per BSN) and several routers. For an efficient solution for channel allocation and mobility management in WHSNs, that cellular architecture, based on UWB/Wifi technologies, is chosen to the third level to have at the end a three-tier hierarchical cellular network. The detailed description of the network architecture is out of scoop of this paper, so for more details, one can refer to [9]. Let us assume the general case of a network composed by $N_{c}$ PANs uniformly distributed. The ideal case of an hexagonal model is chosen to ensure the totality coverage of the network. Although in practice the coverage zone of a sensor device is neither an hexagon nor a perfect circle, there are procedures and mechanisms [10] that ensure the adjustments of the model during network deployment by means of experimental test of measurements. In the following sections we are only interested in the problem of UWB-channels sharing between PANs, seen that the problem of Wifi-channels sharing within a mesh network is already treated in [11].

\section{B. IEEE 802.15.4a IR-UWB Spectrum Resource}

IEEE 802.15.4a IR UWB complaint devices can operate in three independent bands: (1) the sub-gigahertz band (250$750 \mathrm{MHz})$, (2) the low band (3.1-5 GHz) and (3) the high band (6-10.6 GHz) (See Fig. 2). As shown in the table 39d given in [12], we dispose of 16 physical frequency channels associated with 8 sequence codes to have in total 32 logical channels. According to table $39 \mathrm{~d}$ given in [12] and Fig. 2, neither overlapping channels nor adjacent channels share same 
sequence codes. Let us assume that $N_{t c h}$ represents the set of all available logical channels. Conforming to worldwide UWB regulation, $\operatorname{Card}\left(N_{t c h}\right)$ is equal to 32,18 and 22 for respectively US, Europe and Japan region. Although bandwidths of the UWB channels are different the IEEE 802.15.4a UWB physical layer operating with any channel can support all proposed data rates (0.11 Mbps to $27.24 \mathrm{Mbps})$.

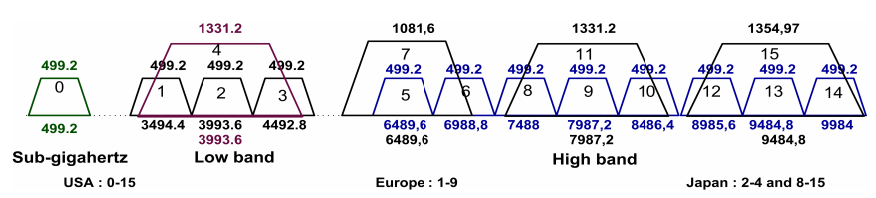

Fig. 2. IEEE 802.15.4a UWB plan bands

According to radio transceiver characteristics, channel switch latency does not exceed $200 \mu s$. Although we can assume that during one duty cycle the additional delay introduced by radio channel switch is not significant, an efficient channel-switch protocol must be proposed to avoid unnecessary channel switches. To switch from a channel to another we need just to firstly program the set of available channel frequencies at a specific register (eg FSCTRL.FREQ for CC2420 transceiver) then to select a given channel. This register must be set to the adequate value.

\section{Static ChanNel ALlOCATION}

\section{A. Allocation of Control Channels}

According to network organization and by means of an optimal coloring algorithm, we try to ensure an efficient control channel allocation. For this, we propose a static allocation of an optimal number of control channels. To avoid the congestion of control channel [6], we propose to statically assign one control channel to each PAN. The emission power density of the UWB signals is less than $-41.3 \mathrm{dBm} / \mathrm{MHz}$ [12]. Given that, overlapping channels $(4,7,11$ and 15) are characterized by high bandwidth [12], they can allow higher transmit power, permitting an extended range, compared to non-overlapping channels. To persistently cover each cell with control traffic, we find that the overlapping channels are more suitable to ensure the zone coverage of such traffic. Taking the simple example of a network of $N_{c}$ cells with a same radius $R$ equals to the PAN coordinator coverage zone that we assume be circular. All PAN members transmit with the same transmitter power $P_{0}$. The choice of $P_{0}$ is done by taking into account Eq.1.

$$
\begin{gathered}
P_{R x}^{c}=P_{0}+P l_{R} \\
P_{R x}^{c}-L i n k_{\text {margin }}^{c}=R x_{\text {sensitivity }}
\end{gathered}
$$

Where:

- $P_{R x}^{c}$ represents received power that must obey to Eq.2,

- $P_{0}$ represents transmitter output power (for control traffic),

- $P l_{R}$ represents path loss at a distance $R$ (cell radius), the expression of path loss is given in [12],
- Link $k_{\text {margin }}$ represents the link margin (for control traffic),

- $R x_{\text {sensitivity }}$ represents the receiver sensitivity.

In this case, all PAN's members precisely those located near the cell border must hear control traffic (beacon frame,.) of their PAN coordinator. Also, PAN coordinator must hear all its members. So, frequency reuse can only occur if the nodes belonging to different PANs are separated by more than 2 hops. This problem can be formulated as 2-hop coloring problem. Consequently, as shown in Fig.3, the minimum distance of frequency reuse must be strictly bigger than 2 hops or distance $R_{c}$ (worst case). Let $D_{\min }$ represents the minimal distance of frequency reuse refereing to cells centers:

$$
D_{\min }>R_{c}=2 \times R
$$

From (3) and according to network hexagonal representation, the shortest distance frequency reuse at cells centers will be:

$$
D_{\text {min }}=4 \times[R(\sqrt{3} / 2)]=(2 \sqrt{3}) R
$$
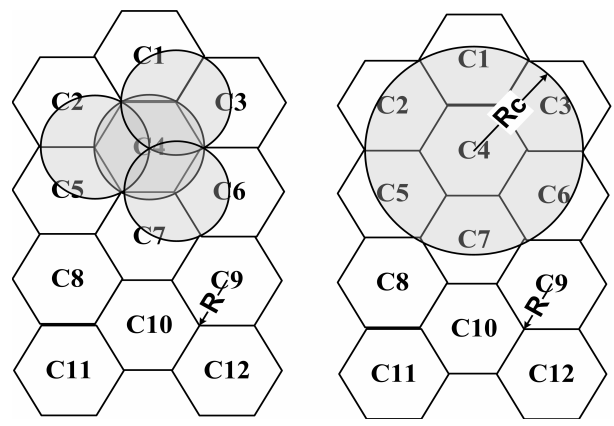

Fig. 3. Radio coverage limit of a logical control channel

In this part, we are interested to find the minimal or optimal number of logical control channels $N_{\text {cch-opt }}$ ensuring a complete network coverage taking into account frequency reuse. This problem can be modeled as graph coloring problem "vertex coloring". As shown in Fig. 4, we can represent our network as a $G(V, E)$ graph, where:

- Each cell center represents a vertex: $V$.

- Distance separating two cells centers that is shorter than $D_{\min }$ represents edge: $E$.

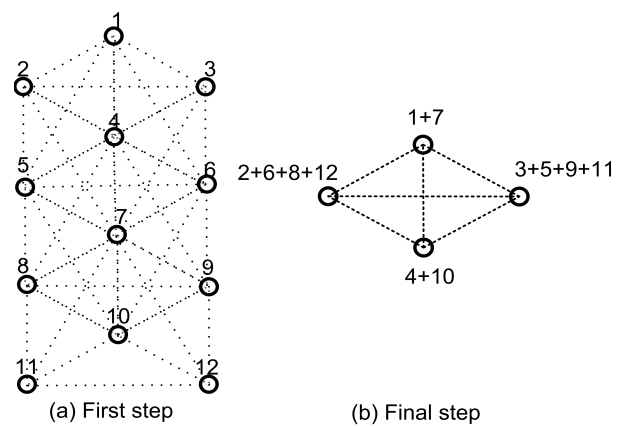

Fig. 4. Logical control channel allocation graph 
According to Fig. 4 no two cells near than $D_{\min }$ share the same control channel. For that we can call for one of optimal coloring algorithms such as Zykov's algorithm, branch and bound method, etc. The application of Zykov's algorithm to previous graph produces nine sub graphs, due to space restrictions; we represent only the final step which is given by Fig. 4.b. In step 9, we are left with a complete graph ("A complete graph with n vertices obviously requires n colors" [13]) with 4 vertices. To cover the entire network by control traffic without suffering from co-channel interference, we just need 4 different channel frequencies. In [14], we propose an analytical proof to generalize this result for networks of more than four adjacent cells.

\section{B. Allocation of Data Channels}

Similar to our approach proposed for control channel allocation, we propose the use of the set of residual channels for data communication but in the context of full mesh network. We assume that all PAN members transmit with the same transmitter power $P_{1}$, in order to have a coverage of radius $r$.

$$
r<R / 2
$$

Given a PAN's mesh topology, we propose a multi-hop routing inside each cell where the hop must be equal to (or shorter than) $r$ in order to:

- decrease transmit power to save sensor battery, maximize network life time and avoid interference,

- balance energy consumption and load over all network cells.

The choice of $P_{1}$ is done by taking into account Eq.6.

$$
\begin{gathered}
P_{R x}^{d}=P_{1}+P l_{r} \\
P_{R x}^{d}-L i n k_{\text {margin }}^{d}=R x_{\text {sensitivity }}
\end{gathered}
$$

Where:

- $P_{R x}^{d}$ represents received power that must obey to Eq.7,

- $P_{1}$ represents transmitter output power (for data traffic),

- $P l_{r}$ represents path loss at a distance $r$,

- Link margin $^{d}$ represents the link margin (for data traffic).

As shown in Fig.5, taking the example of the $4^{t h}$ cell, we note that sensors located at or near the cell border can interfere with sensors of adjacent PANs, located at or near their cell border. Consequently, as shown in Fig. 5, the minimum distance of frequency reuse must be strictly bigger than $R_{c}$ (worst case). Let $D_{\min }^{\prime}$ represents the minimal distance of frequency reuse refereing to cells centers:

$$
\begin{aligned}
& D_{\text {min }}^{\prime}>R_{c} \\
& R_{c}=R+r
\end{aligned}
$$

From (8), (9) and according to network hexagonal representation, the shortest distance frequency reuse at cells centers will be :

$$
D_{\min }^{\prime}=3 \times R
$$

In this part, we are interested to find the minimal number of data communication channels $N_{d c h-o p t}$ to cover the global network taking into account frequency reuse. Similar to the case of control channel allocation, this problem can be translated into graph coloring problem applied to the graph $G^{\prime}(V, E)$ shown by Fig. 6, such that no two adjacent vertices share the same color. The application of Zykov's algorithm to graph $G^{\prime}(V, E)$ produces ten sub graphs. In the $10^{\text {th }}$ step given by Fig. 6.b, we are left with a complete graph. The optimal solution is given by the complete graph with three vertices (ie $N_{d c h-o p t}=3$ ). To cover the entire network from communication traffic without suffering from co-channel interference, we just need 3 different channel frequencies. In [14], we propose an analytical proof to generalize this result for networks of more than three adjacent cells.

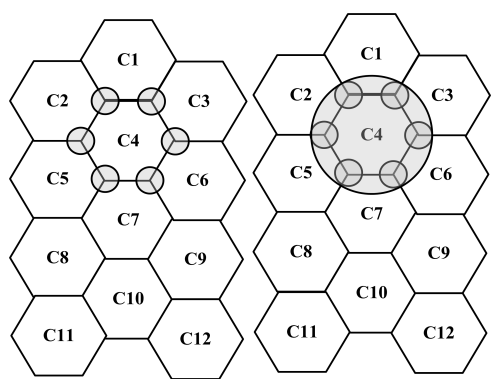

Fig. 5. Radio coverage limit of a logical data communication channel

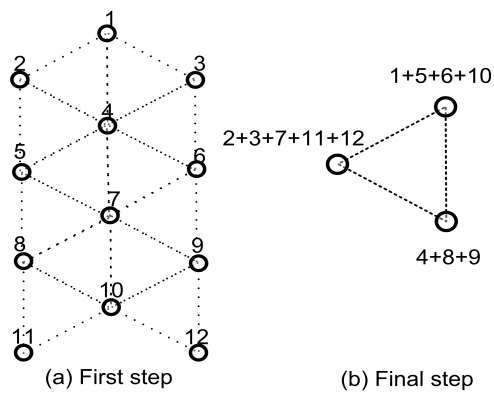

Fig. 6. Logical data communication channel allocation graph

\section{DYNAMIC DATA CHANNEL ALLOCATION}

To ensure dynamic data channel allocation, our approach consists in taking advantage of data channel allocation method, previously detailed, but with the exploitation of the duty cycle's information of PANs. According to available data channel frequencies $N_{d c h}$ and PANs duty cycle, each PAN can benefit simultaneously from several data channels.

We define $K$ as :

$$
K=\operatorname{ceil}\left[\operatorname{Card}\left(N_{d c h}\right) \operatorname{Div} N_{d c h-o p t}\right]
$$

$K$ represents the number of simultaneous data channel frequencies that can benefit each PAN. From a spectrum regulation to another, $K$ isn't the same, for US: $K=8$, Europe: $K=4$ and Japan: $K=6$. But in reality each 
PAN is characterized by its duty cycle or superframe duration, as shown in Fig. 7. So, according to PANs duty cycle, $K$ can change during global network active period. Let us assume the general case of a network composed by $N_{c}$ PAN coordinators with correspondent superframe durations $\left\{P A N_{i}=\left(S D_{i}, B I_{i}\right)\right\}_{1 \leq i \leq N_{c}}$. We define $\overline{B I_{m a j}}, \overline{S D_{\min }}$ and $U$ as respectively the major cycle, the elementary active cycle (ie elementary time unit) and the number of elementary active cycle per major cycle.

$$
\begin{gathered}
\overline{B I_{m a j}}=L C M\left(B I_{1}, B I_{2}, \ldots, B I_{N_{c}}\right) \\
\overline{B I_{m a j}}=L C M\left(2^{B O_{1}}, 2^{B O_{2}}, \ldots, 2^{B O_{N_{c}}}\right) \\
\overline{B I_{m a j}}=\max _{1 \leq i \leq N_{c}}\left(2^{B O_{i}}\right) \\
\overline{S D_{\min }}=L C D\left(S D_{1}, S D_{2}, \ldots, S D_{N_{c}}\right) \\
\overline{S D_{\min }}=L C D\left(2^{S O_{1}}, 2^{S O_{2}}, \ldots, 2^{S O_{N_{c}}}\right) \\
\overline{S D_{\min }}=\min _{1 \leq i \leq N_{c}}\left(2^{S O_{i}}\right) \\
U=\frac{\overline{B I_{\operatorname{maj}}}}{\overline{S D_{\min }}}
\end{gathered}
$$

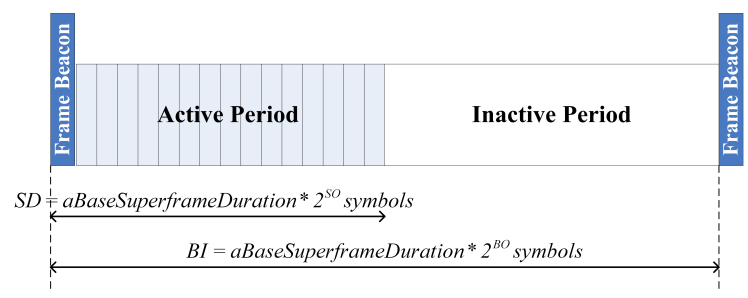

Fig. 7. PAN superframe structure

Let $D C_{N_{c} x U}$ and $D_{N_{c} x N_{c}}$ represent respectively the matrix of duty cycle of all PANs coordinators per elementary active cycle and the matrix of distances separating the centers of each couple of cells.

$$
\begin{aligned}
D C_{N_{c} x U} & =\left(\begin{array}{cccc}
D C_{11} & . . & . . & D C_{1 U} \\
D C_{21} & . & . & . . \\
. . & . . & . . & D C_{N_{c}-1 U} \\
D C_{N_{c} 1} & . . & . . & D C_{N_{c} U}
\end{array}\right) \\
D_{N_{c} x N_{c}} & =\left(\begin{array}{cccc}
0 & . . & . & D_{1 N_{c}} \\
D_{21} & 0 & . . & . . \\
. . & . . & 0 & D_{N_{c}-1 N_{c}-1} \\
D_{N_{c} 1} & . . & . . & 0
\end{array}\right)
\end{aligned}
$$

Given $D C_{N_{c} x U}, D_{\min }^{\prime}$ and $D_{N_{c} x N_{c}}$, we can determine the graph $G^{\prime}(V, E)_{i}$ per elementary active cycle and then compute optimal number of data channels to cover entire network as done in previous section (worst case where all PAN coordinators are active). In last step, we compute the number of simultaneous data communication channels $K_{i}$ per active PAN for the relative elementary active cycle.

$$
K_{i}=\operatorname{ceil}\left[\operatorname{Card}\left(N_{d c h}\right) \operatorname{Div} N_{d c h-o p t_{i}}\right]
$$

In conclusion, considering the available data communication channels $N_{d c h}, D C_{N_{c} x U}, D_{N_{c} x N_{c}}$ and $D_{m i n}^{\prime}$, we can compute the matrix of sub set of data channels per PAN per elementary active cycle. For example $N_{d c h_{11}}$ represents the set of data channels used by the first PAN (ID =1) during the first elementary active cycle, where $\operatorname{Card}\left[N_{d c h_{11}}\right]=K_{i}$.

$$
N_{d c h_{N_{c} x U}}=\left(\begin{array}{cccc}
N_{d c h_{11}} & . . & . . & N_{d c h_{1 U}} \\
N_{d c h_{21}} & . . & . . & . . \\
. . & . & . & N_{d c h_{N_{c}-1 U}} \\
N_{d c h_{N_{c} 1}} & . . & . . & N_{d c h_{N_{c} U}}
\end{array}\right)
$$

\section{Performance evaluation}

We have implemented the proposed schemes in a discretetime simulator built in JAVA and based on some functionalities defined by Prowler simulator [15] with the support of the network architecture proposed in [9]. For static allocation our algorithm presents a complexity of the order $O(n)$, where for dynamic allocation it is less fast and it presents a complexity of the order $O\left(n^{2}\right)$. For the rest of the paper, we refer to our channel allocation scheme by UWBCAS (Ultra Wide Band Channel Allocation Scheme)

\section{A. Simulation Scenario}

Let us consider a synchronized UWB-based WHSN of 9 PANs with radius of $5 \mathrm{~m}$. Each PAN has 35 nodes uniformly distributed (One PAN coordinator, thirty routers and four BSNs coordinators). Taking the example of the worst case given by Fig. 8 where all PANs begin communication at the same time. Let us assume that the European regulation is adopted (ie channels 4 and 7 for control and the rest for data). For each active PAN, we assume that six nodes initiate CBR streams towards the sink nodes and each source node generates a packet in every time slot. To eliminate the bottleneck problem of single sink node, we assume that there are several sink nodes in each PAN. Each $P A N_{i}$ is characterized by its superframe duration $\left(S D_{i}, B I_{i}\right)$ as shown by Fig. 8. So, $\overline{B I_{m a j}}=32, \overline{S D_{\min }}=1, U=32$.

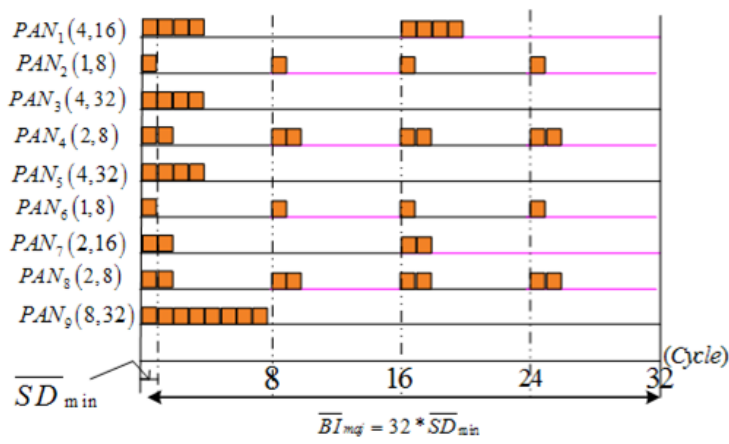

Fig. 8. Example of PAN configuration

TABLE I shows the default value of each parameter in the simulations. 
TABLE I

SIMULATION PARAMETER

\begin{tabular}{|c|c|}
\hline Parameter & Default value \\
\hline Number of PANs & 9 \\
\hline PAN's radius & $5 m$ \\
\hline $\begin{array}{c}\text { Number of Nodes } \\
\text { per PAN }\end{array}$ & $\begin{array}{c}35: \text { (1 PAN coordinator, } \\
4 \text { BSNs, } 30 \text { Routers })\end{array}$ \\
\hline Node placement & Uniform \\
\hline Communication rate & $850 K b p s$ \\
\hline Radio range & $5 m$ for control, $2 m$ for data \\
\hline Data Packet length & 127Bytes \\
\hline System Load & 6 packet/PAN/Time slot \\
\hline Time slot duration & $0.985 \mathrm{~ms}$ \\
\hline$S O_{\min }$ & $\begin{array}{c}4,(P A N \text { 's superframe Order } \\
\left.\text { will be normalized by } S O_{\min }\right)\end{array}$ \\
\hline Network Duty Cycle & See Fig. 8 \\
\hline
\end{tabular}

We conduct simulation with the configuration given in TABLE I. We obtain a data channel distribution as illustrated in Fig. 9, where:

- During the $1^{\text {st }}, 2^{\text {nd }}$ and $17^{\text {th }}$ elementary cycles, each active PAN benefits simultaneously from 4 channels.

- During the $3^{r d}, 4^{t h}, 9^{t h}, 18^{t h}$ and $25^{t h}$ elementary cycles each active PAN benefits from 7 channels (complete graph is composed by two vertices).

- During the $5^{t h}, 6^{t h}, 7^{t h}, 8^{t h}, 19^{t h}$ and $20^{t h}$ elementary cycles, only one PAN ( $1^{\text {st }}$ or $\left.9^{t h}\right)$ is active which benefits simultaneously from all available channels.

- During the $10^{t h}$ and $26^{t h}$ elementary cycles only two PANs $\left(4^{\text {th }}\right.$ and $\left.8^{\text {th }}\right)$ are active, each one benefits simultaneously from all available channels because the distance separating those two PANs is greater than $D_{m i n}^{\prime}$.

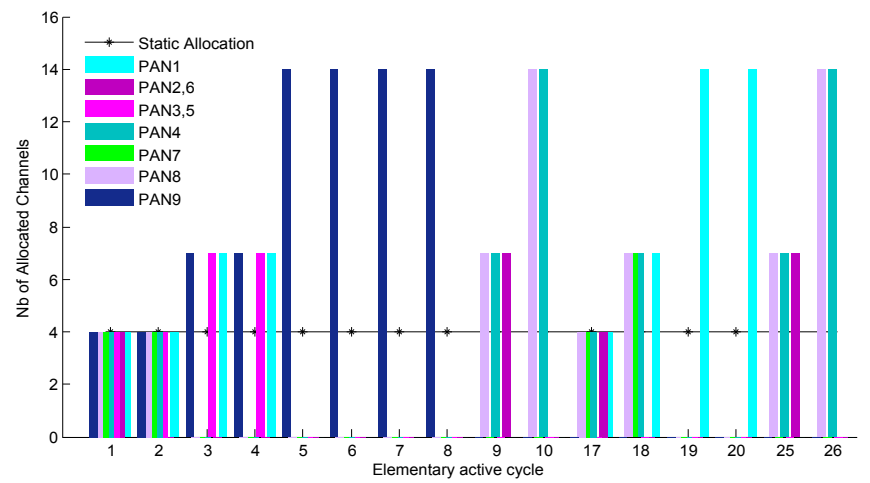

Fig. 9. Data channel allocation during active elementary cycles

Fig. 10 illustrates the results obtained with static and dynamic data channel allocation. The simulation is conducted with different spectrum regulations as well. We note that with static data channel allocation, the maximum number of allocated channels per PAN is 8, 6 and 4, respectively for US, Japanese and European regulation. Where with dynamic data channel allocation, during given elementary cycles, active PANs can benefit from supplementary channels which are initially been allocated to some other PANs.

During the $5^{t h}, 6^{t h}, 7^{t h}, 8^{t h}, 10^{t h}, 19^{t h}, 20^{t h}$ and $26^{t h}$, active PANs benefit up to 28,18 and 14 in respectively US, Japanese and European regulation.

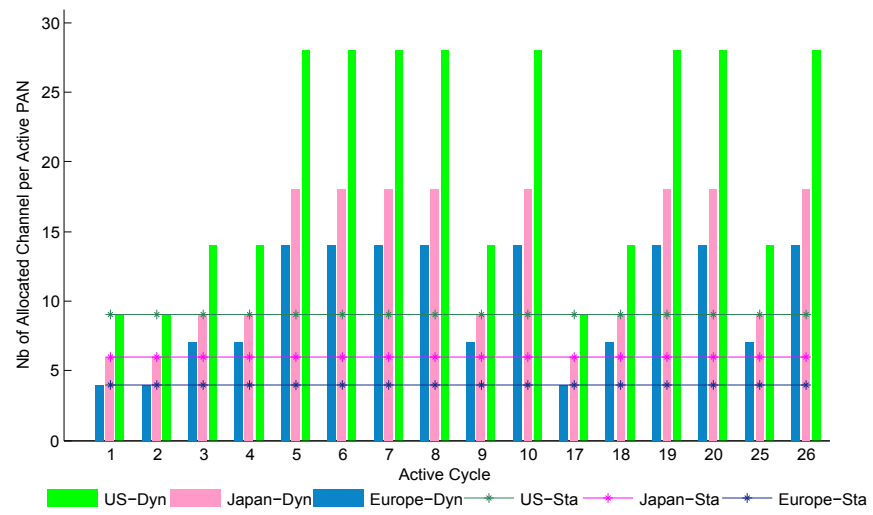

Fig. 10. Static vs Dynamic channel allocation

\section{B. Comparative Performance Evaluation}

In this part, we conduct simulations to compare UWBCAS, on the one hand, with MCMAC [7], characterized by a complexity of the order $O(n)$, in terms of channel utility, throughput and delay and, on the other hand, with MMSN [4], characterized by a complexity of the order $O\left(n^{2}\right)$, in terms of required number of channels to enable the network operation.

1) Channel Utility: We define the Channel Utility H $_{j}$ as the ratio of the sum of channel frequencies used by each active PAN per available data channel frequencies (of the $j^{\text {th }}$ elementary cycle).

$$
\text { Channel Utility }_{j}=\frac{\sum_{i=0}^{N_{\text {active }_{j}}} N_{d c h_{i j}}}{N_{d c h}}
$$

where $N_{\text {active }_{j}}$ represents the number of active PAN during the $j^{\text {th }}$ elementary cycle, with $N_{\text {active }_{j}} \leq N_{c}$.

In this part, we compare the behavior of each scheme in terms of channel utility for the same amount of load. As shown in Fig. 11, the MCMAC presents a constant channel utility percentage given that it allows only one PAN to be active. With UWBCAS, from an active cycle to another, results vary according to the variation of the number of both active PANs and allocated channels per active PAN. It is observed that UWBCAS always exhibits better performance than MCMAC because it allows several PANs to communicate simultaneously. This can ensure the full use of available resource. 


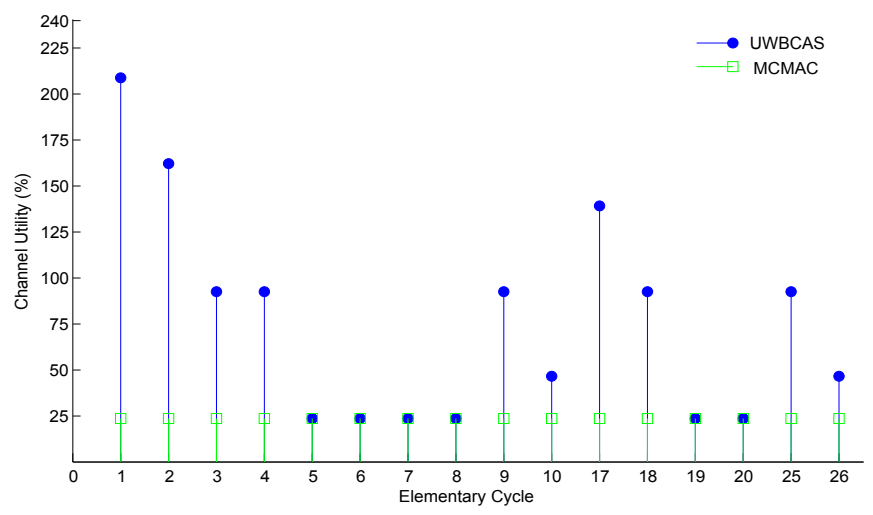

Fig. 11. Channel Utility vs Time

Fig. 12 highlights the behavior of UWBCAS in terms of channel utility through the whole network by varying the system load. We note that the channel utility increases with the increase of the system load and it can increase more when the number of active PANs increases. Almost in every case, we discover that the channel utility performance of UWBCAS is better than that of MCMAC. Fig. 12 shows that, with all system loads, our protocol becomes more spectrum efficient when the number of active PAN are important which is consistent with the result presented in the previous experiment.

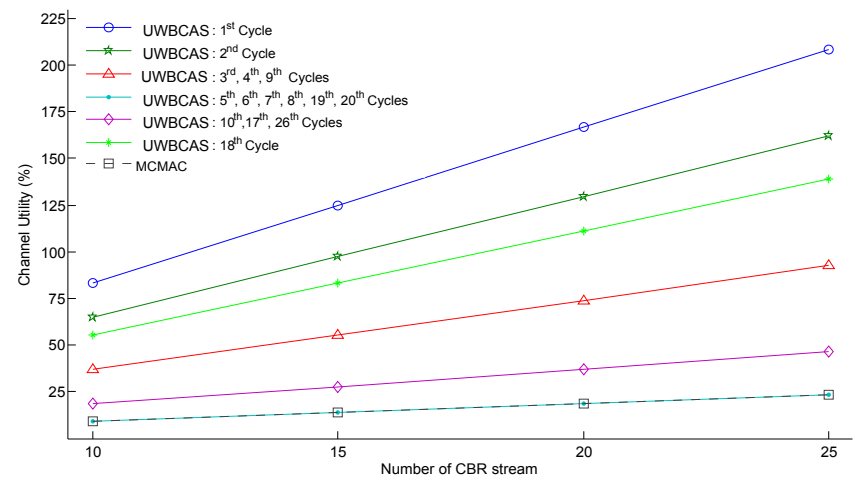

Fig. 12. Channel Utility vs System Loads Variety

2) Throughput: We take the global throughput as a metric to measure schemes performance. Fig. 13 shows that, MCMAC presents almost a static behavior where UWBCAS offers better results. Once again, UWBCAS makes its proof to guarantee the enhancement of the throughput of the global network. This guarantee comes from the exploitation of the dynamic data channel allocation policy based on PANs duty cycle's information that avoids the underutilization of spectrum resource and maximizes the number of used channels per active PAN.

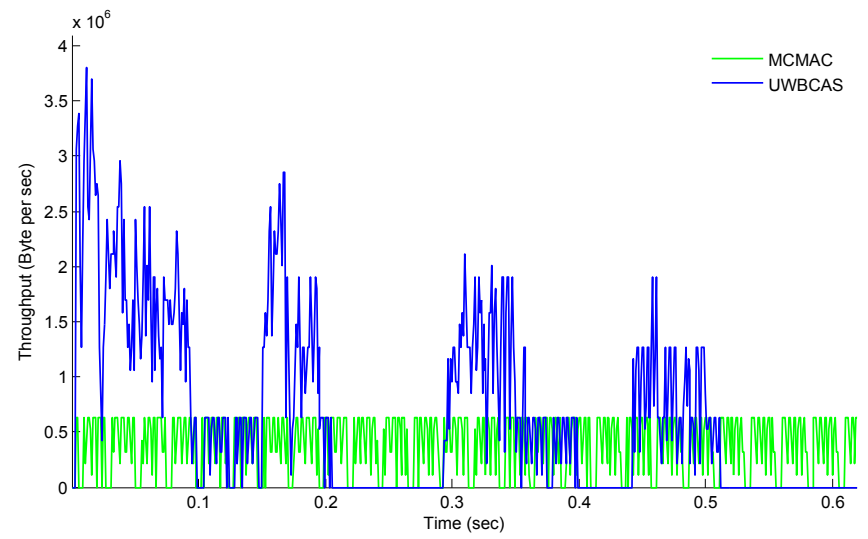

Fig. 13. Throughput vs Time

Although globally our model offers the best result but if we analyze the behavior of a single PAN per time slot we find that MCMAC presents a slightly enhanced result as shown in Fig. 14. With MCMAC, given that there are only one active PAN per cycle all available channels will be allocated to it. This can locally enhance PAN's performance during given time slots but not globally for the entire network.

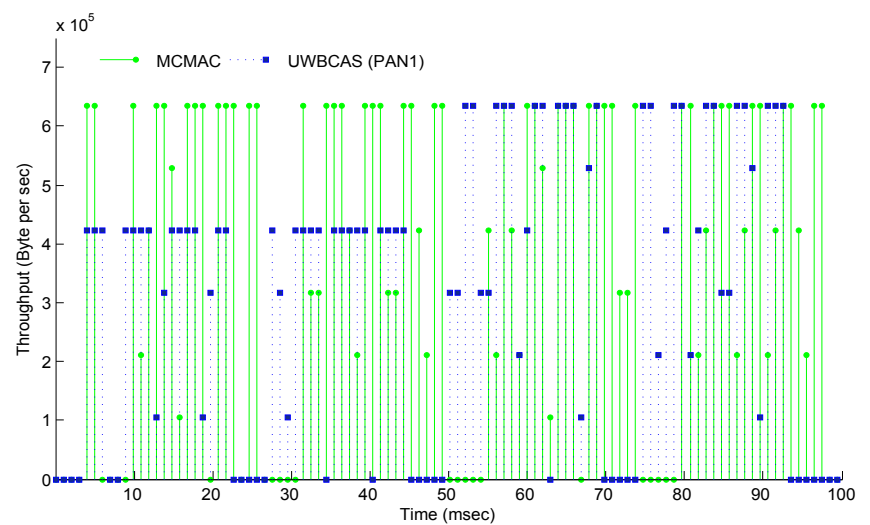

Fig. 14. Throughput vs Time of the first PAN

3) Delay: We define the average end-to-end delay per packet as the ratio of the sum of delay $\left(\delta_{i}\right)$ experienced by each packet making up the flow per number of packets $(N)$.

$$
A V G(\text { End2End Delay per packet })=\frac{\sum_{i=0}^{N} \delta_{i}}{N}
$$

We propose to evaluate the behavior of both schemes in terms of the average end-to-end delay per packet. Fig. 15 shows that UWBCAS always exhibits better performance than MCMAC. With UWBCAS, the decrease of the end to end delay is mainly due, on the one hand, to the increase of the number of simultaneous communications and, on the other hand, to the avoidance of the phenomena of congestion (data and control traffic). 


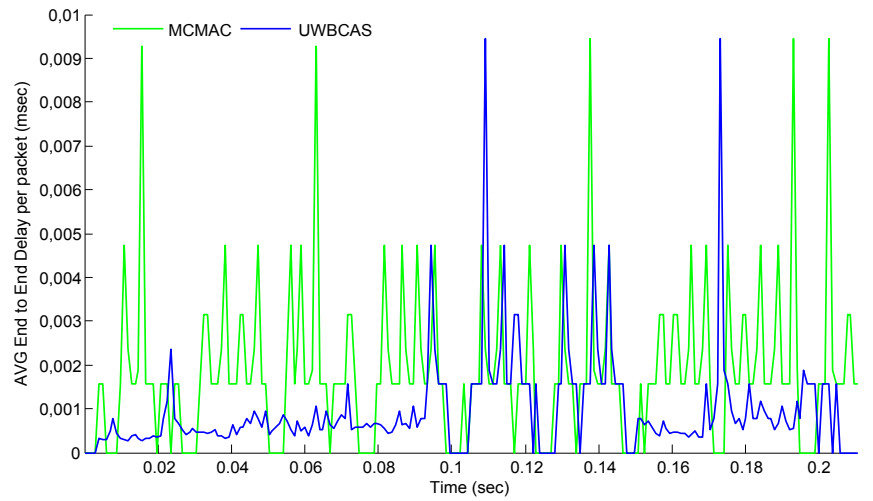

Fig. 15. Delay vs Time

4) UWBCAS vs MMSN: Taking the case of the first scheme proposed by MMSN (exclusive frequency assignment scheme) and UWBCAS, we propose to give an idea about the number of required channels, to enable the operation of the network, for different node densities. The node density is increased from 6 to 21 by configuring different radio ranges (from 1.5 to $3.5 \mathrm{~m}$ ). Fig. 16 mentions that the behavior of exclusive frequency assignment scheme is considerably sensitive to the node density factor. The number of required channels increases when the node density increases. Contrarily to MMSN, with UWBCAS the operation of the network doesn't depend on the node density factor. We need at most seven different channel frequencies to launch the network then to improve its performance the use of more channel frequencies is encouraged. Compared to UWBCAS, exclusive frequency assignment scheme can not ensure an efficient use of spectrum resource given that it allocates channels in static way that limits channel utility as a node does not permanently use its assigned channel.

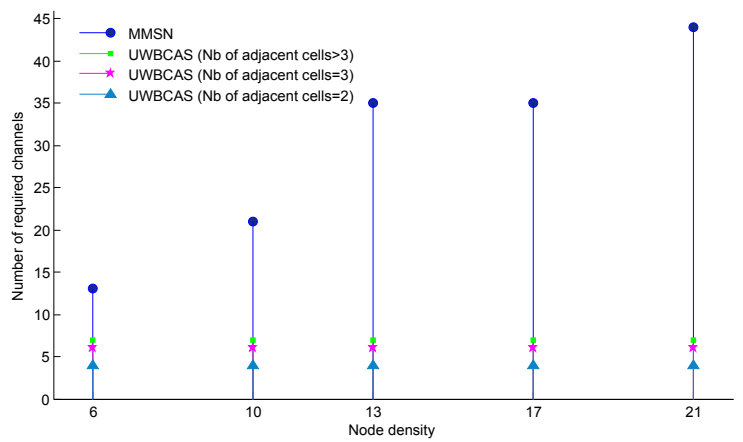

Fig. 16. Required channels in UWBCAS and MMSN in function of node density

\section{CONCLUSION}

In this paper, we presented frequency allocation schemes for an efficient spectrum management, in large-scale and dense WSNs. We have decomposed the frequency allocation problem into two sub-problems: static control channel allocation to ensure a permanent control channel frequency per PAN avoiding the problem of control channel congestion and dynamic data channel allocation based on PANs duty cycle's information and spatial frequency reuse to avoid the underutilization of spectrum resource. We implemented our schemes on in a discrete-time simulator built in JAVA. Evaluation results demonstrate that our schemes come to reach our goals in terms of spectrum efficiency which under an adequate multichannel MAC protocol can significantly improve network performances. In this way, we can ensure, on the one hand, an efficient and fair data channel allocation between PANs permitting an enhancement of QoS of the entire network and, on the other hand, a maximization of the channel utility. As continuation of this work, we propose in future a prioritized multi-channel multi-time slot MAC protocol for Large-Scale WSNs.

\section{REFERENCES}

[1] X. Wang and T. Berger, "Spatial channel reuse in wireless sensor networks", Wireless Networks Journal, vol 14, pp. 133-146, March 2008.

[2] CC2420 datasheet, 2004 chipcon, inst.eecs.berkeley.edu/ cs150/Documents/CC2420.pdf

[3] X. Lagrange, P. Godlewski and S. Tabbane, "Reseaux GSM-DCS ", Herms Sciences Publications, 1999.

[4] G. Zhou, C. Huang, T. Yan, T. He and J. Stankovic, A. Abdelzaher, "MMSN: multi-frequency media access control for wireless sensor networks", INFOCOM 2006. 25th IEEE International Conference on Computer Communications Proceedings, April 2006, pp. 1 - 13.

[5] R.E. Cagley, S.A. McNally and M.R. Wiatt, "Dynamic channel allocation for dynamic spectrum use in wireless sensor networks" Military Communications Conference, October 2006, pp. 1-5.

[6] H. So, W. Walrand and J. Jeonghoon, "McMAC: a parallel rendezvous multi-channel MAC protocol", IEEE Wireless Communications and Networking Conference, March 2007, pp. 334-339.

[7] X. Chen, P. Han, Q. He, S. Tu and Z. Chen,"A multi-channel MAC protocol for wireless sensor networks", The 16th IEEE International Conference on Computer and Information, 2006, pp.224-229.

[8] A. Rangnekar, K.M. Sivalingam, "Multiple Channel Scheduling in UWB based IEEE 802.15.3 Networks", Proceedings of the First International Conference on Broadband Networks, October 2004, pp.406-415.

[9] J. Ben Slimane, Y.Q .Song, A. Koubaa, M. Frikha, "A Three-Tiered Architecture for Large-Scale Wireless Hospital Sensor Networks", the International Workshop on Mobilizing Health Information to Support Healthcare-Related Knowledge Work - MobiHealthInf 2009, pp 20-31.

[10] J. Jemai, R.Piesiewicz, T.Kurner, "Calibration of an indoor radio propagation prediction model at $2.4 \mathrm{GHz}$ by measurements of the IEEE $802.11 \mathrm{~b}$ preamble" IEEE 61st Vehicular Technology Conference, Spring. 2005,Vol 1, pp.111-115.

[11] B. Raman, "Channel Allocation in 802.11-Based Mesh Networks", 25th IEEE International Conference on Computer Communications, INFOCOM 2006, pp. 1-10.

[12] IEEE 802.15.4a Standard Part 15.4: IEEE Standard for Information Technology, Amendment to IEEE Std 802.15.4-2006, 2007.

[13] A. Kathryn, "Classical Techniques", Springer US Book, chapter 2, 2005, pp.19-68.

[14] J. Ben Slimane, Y.Q .Song, A. Koubaa, M. Frikha, "Allocation of control and data channels for Large-Scale Wireless Sensor Networks", Technical report, 2008, http://hal.inria.fr/inria-00322584/fr/.

[15] http://www.isis.vanderbilt.edu/projects/nest/prowler/. 